\title{
THE
}

\section{Clinician Perceptions of the NICU Infant Experience: Is the NICU Hospitalization Traumatic?}

Amy L. D'Agata

University of Rhode Island, amydagata@uri.edu

Mary Coughlin

Marilyn R. Sanders

Follow this and additional works at: https://digitalcommons.uri.edu/nursing_facpubs

\section{The University of Rhode Island Faculty have made this article openly available.} Please let us know how Open Access to this research benefits you.

This is a pre-publication author manuscript of the final, published article.

Terms of Use

This article is made available under the terms and conditions applicable towards Open Access Policy Articles, as set forth in our Terms of Use.

\section{Citation/Publisher Attribution}

D'Agata, A. L., Coughlin, M., \& Sanders, M. R. (2018). Clinician Perceptions of the NICU Infant Experience: Is the NICU Hospitalization Traumatic? Am J Perinatol. 2018, 35(12), 1159-1167. doi: 10.1055/ s-0038-1641747 Available at: http://dx.doi.org/10.1055/s-0038-1641747 


\title{
Clinician Perceptions of the NICU Infant Experience: Is the NICU Hospitalization Traumatic?
}

\author{
Amy L. D’Agata, PhD, MS, RN ${ }^{1}$ \\ Assistant Professor \\ Mary Coughlin, BSN, RN, MS, NNP, RNC-E ${ }^{2}$ \\ Educator and Consultant \\ Marilyn R. Sanders, MD, FAAP \\ Attending Neonatologist ${ }^{3}$ and Professor ${ }^{4}$
}

1- College of Nursing, University of Rhode Island, Kingston, RI

2- Caring Essentials Collaborative, LLC, Boston, MA

3- Connecticut Children's Medical Center, Hartford, CT

4- School of Medicine, Department of Pediatrics, University of Connecticut, Farmington, CT

Corresponding author:

Amy D'Agata

350 Eddy Street, Providence, RI 02903

401-874-9703

amydagata@uri.edu

Conflict of interest:

Drs. D'Agata and Sanders have no conflicts of interest relevant to this article to disclose; Mary Coughlin is the President and Founder of Caring Essentials Collaborative, LLC.

The authors have no financial relationships relevant to this article to disclose.

Human subject approval was obtained for this work. 


\begin{abstract}
Objectives: Infants cared for in a newborn intensive care unit (NICU) experience pain, parental separation and stress that may approach toxic levels, thus are potentially traumatic. Lack of accepted clinical terminology to describe the infant experience may result in under appreciation of NICU hospitalization on infant and family outcomes. This study explored NICU clinician perceptions of the infant experience and how the terms trauma/traumatic would impact their clinical roles and practices.
\end{abstract}

Study Design: Semi-structured focus group interviews and thematic analysis were used to describe professionals' perceptions of the infant's experience and terminology. Focus groups were organized by professional role, including NICU leadership, physicians, nurses and ancillary providers.

Result: Six themes emerged from the qualitative analysis: at our mercy; trauma defined and redefined; and now you have broken them too; perceptions of NICU experience change over time; trauma in the NICU: whose trauma is it; not knowing the infant and family experience.

Conclusion: While recognizing potentially toxic infant stress levels, clinicians are reluctant to describe the NICU infant experience as traumatic. Hesitations relate to clinicians' personal concerns that they may be seen as agents of trauma and the impact for families if the NICU experience was described as traumatic by clinicians.

Key words: preterm infants, NICU, trauma, traumatic, focus group 


\section{INTRODUCTION}

"Children reflect the world in which they are raised." 1

The impact of adverse childhood events (ACEs) on later health and development now informs the science of early experience by addressing the implications of stressful exposures in childhood $^{2}$. The National Scientific Council on the Developing Child (NSCDC) distinguishes between positive, tolerable, and toxic stresses ${ }^{3}$. Positive, or short-lived stress responses, as a normal part of healthy development, teach the infant and then child to self-soothe and selfregulate. Tolerable stresses occur in the context of an ongoing supportive relationship with caring adults who assist the infant/child in self-regulation. Toxic stress without supportive, caring relationships derails healthy development through excessive and prolonged activation of stress responses, including hypothalamic-pituitary-adrenal axis and sympathetic nervous system activation ${ }^{3}$. In the absence of supportive caregiving, the infant/child also fails to receive appropriate positive stimulation for brain growth and development ${ }^{4}$.

The National Traumatic Children's Stress Network, based upon parent report, recently designated medical illness, a traumatic stress in childhood ${ }^{5}$. Newborn intensive care hospitalization results in violations of newborn biological expectancies, including the physical and emotional proximity of their co-regulatory caregivers. Coughlin and the National Perinatal Association recognizing that life threatening illness, hospitalization, and separation from mother and family may elicit toxic stress responses call for implementation of trauma-informed care principles in the NICU ${ }^{6}$.

D’Agata et al (2016) proposed a conceptual model for infant medical trauma in the NICU (IMTN) that facilitates an interdisciplinary approach for studying the infant's experience, including the inextricably bound effects of stress, parental separation and pain ${ }^{7,8}$. The IMTN 
model and the recent work of Coughlin (2016) acknowledge the potentially traumatic experience of the NICU infant and family. Together, they suggest implementation of evidence-based trauma-informed NICU care may mitigate potentially traumatic relational disruptions for the infant, the family and the clinician. Infusing this paradigm into clinical work, however, requires insight into the clinicians' understanding of the infant's experience of care in the NICU ${ }^{8,9}$.

The purpose of this qualitative analysis was to explore terminology that describes the NICU infant experience, through focus group discussions with neonatal healthcare providers. The specific research questions asked: 1) what terminology best describes neonatal caregivers' perceptions of the early life experience of infants cared for in the NICU, and 2) do neonatal caregivers perceive the NICU infant experience as traumatic?

\section{PARTICIPANTS and METHODS}

This study used a focus group design to study clinician perceptions of terminology to describe the NICU infant experience. The selection of setting and sample were purposive. The study was conducted in a level IV NICU, located in a Northeastern perinatal/neonatal regional academic center, from October to December 2015. Institutional Review Board approval was granted by the participating hospital.

To encourage diversity of perspectives and perceptions in the focus group discussions, all neonatal intensive care nurses, attending neonatologists, neonatology fellows, neonatal nurse practitioners, physician assistants, respiratory therapists, social workers, lactation consultants, dieticians, nursing educators, assistant and nurse managers, and NICU directors were invited to participate. A letter of invitation was sent by email, flyers were posted for staff and informal discussions of the study purpose occurred with sample stakeholder groups. Clinicians' eligible to participate included those employed at least part-time in the NICU and with a minimum of one- 
year professional NICU work experience. Participants provided written informed consent and a demographic form.

Participants attended a single one-hour focus group, conducted by the first-named author with experience in focus group facilitation. To support open dialogue, the 4 focus groups were organized by professional role: 1) registered nurses; 2) nurse practitioners, physician assistants and attending neonatologists; 3 ) respiratory therapists, lactation consultants and occupational/ physical therapists; 4) assistant and nurse managers, nursing and medical directors. Attendance per focus group ranged from 4 - 8 participants ${ }^{10}$. Table 1 describes the sample of participants by discipline, including 10 nurses, 5 physicians and 2 ancillary clinicians (Table 1). Since neither mathematical formulas nor agreed upon criteria to justify data saturation exist, we determined data saturation to be reached when new participant information was no longer expressed.

Qualitative data were gathered through focus group interviews. Focus groups are a series of carefully planned interviews that allow data to be compared and contrasted across groups ${ }^{10}$. A semi-structured interview format was employed. This approach was chosen to foster deep reflection and organic discussion among NICU providers that may not occur with individual interviews. Focus groups were conducted in a quiet room to minimize interruptions. Following informed consent, a detailed description of the study and confidentiality commitment were provided to participants at the commencement of each focus group. Ten questions designed to guide the discussion of participant perceptions and experiences were developed by the researchers (Table 2). The sessions were audio-recorded and professionally transcribed verbatim; field notes were also generated during the focus groups. Identifying participant information was removed during transcription. 
Inductive thematic analysis was used to analyze the qualitative data. Since the purpose of this investigation was to gain knowledge about a novel topic, an inductive process was selected to identify patterns in this data-drive approach. Thematic analysis is defined as a "method for identifying, analyzing and reporting patterns (themes) within data" ${ }^{11}$. To conduct the thematic analysis we chose the six-step process described by Braun and Clarke (2006): data familiarization, generating initial codes, searching for themes, reviewing themes, defining and naming themes and producing the report ${ }^{11}$.

Following transcription, the three investigators independently reviewed the four focus group transcripts to become familiar with the data. Individually investigators first began the coding process by underlining key phrases in the data and then scrutinizing for patterns across the data to develop initial categories. All investigators then met to discuss and compare key phrases and categories, compare initial codes and generate new codes. The investigators next organized substantive categories of codes based on their relationship to one another, across the interview transcripts, and continued to identify similarities and differences among the data. Several meetings were held to achieve consensus in the data analysis. Finally, the investigators collapsed the items and refined the code into themes, see Table 3. Investigators sought internal coherence in each theme and strong distinctions between themes. Participant quotes were selected that captured the essence of the theme.

Rigor was addressed through credibility, confirmability, dependability and transferability. Having an interdisciplinary nursing and medical research team supported investigator triangulation as the transcripts of audio-recording were analyzed and considered from different professional viewpoints. Multiple coders also supported trustworthiness of the data. In this process, discussion among investigators supplemented or challenged the data analysis, but 
ultimately supported confirmability. The richness of participant quotes was used to describe themes, thereby enhancing transferability. An audit trail of data analysis was maintained for purposes of dependability.

\section{RESULTS}

Insert Table 2 data as results

Thematic analysis revealed six themes from the interviews.

\section{At Our Mercy}

Participants described concerns that vulnerable infants in the NICU have multiple providers, are in an over-stimulating environment, are told when to eat, are touched all the time and suffer significant pain and stress. Procedures and care are done to infants who have no ability to assent or consent. Providers perceived newborns as helpless patients who cannot refuse care and who are fearful.

I picture it being like a blind person; not knowing what's coming at you. You can hear things but what does that mean? $(\mathrm{RN})$

Sometimes there is a way to kind of support them while you are doing it but sometimes there's no time or the person doesn't have any other support person, so they're focused on just doing what they need to do- get that blood- you know, and so the baby may be flailing and crying and screaming, and we are just focused on getting that little thing. It's like you know, this whole assault on the baby in a way. (MD)

What comes to my mind is that they're at our mercy...it's all about what we do and how we approach the situation..., so anybody even a little bit older [than an infant] has some way to express themselves or just stand up for themselves literally. So they really are at our mercy. $(\mathrm{RN})$

We've all experienced the days where you almost just want to run and put your head in a corner because it's just...there's just stuff going on everywhere. Bells and whistles and alarms and beeping and people....ah! And it's like I can't image what these little babies are feeling like... (MD)

\section{2. “Trauma” Defined and Redefined}


Participants defined "trauma" as an experience that is painful, unforgettable, physical and emotional and unexpected. Participants considered how the word "trauma" may sensitize the staff and administration. Potential benefits of using the word "trauma" included improvements in care, potential increased awareness of infant's lived experience, enhanced level of compassion for the infant and the potential to generate additional resources. On the other hand, participants were concerned about the implications of labeling the infant experience as traumatic both for their own duty to their patients and for vulnerable families.

Defined

Stress can eventually make you stronger in an area, where a traumatic event, again, physical or emotional, can actually make you weaker for potentially ever. (MD)

I used to see it as an event only but... now I see it more as an event with all the repercussions of that event- not just on that person, but also on anyone dealing with them... (Respiratory therapist)

Trauma, to me, is like war. You know what I mean? ... I don't want to say that is the environment here in the NICU. You know in some ways it is, because we do inflict pain but it's for a whole different purpose. You know what I mean? That mentality isn't the same as going to war... we're fighting, we're battling to save the babies lives... but ... to me, traumatic is just a severe word. (RN)

When you say trauma you think rape, tsunami, World Trade Center... (RN)

\section{Redefined}

I just think we haven't really ever really used that word in relation to what we do. I think as we've sat here and kind of talked about that, that it makes perfectly good sense that that is what it is- it's a traumatic experience- but we've just never defined it that way before. I think... it just kind of makes you think about things a little bit differently. (RN)

...would you let a baby cry in a crib or wherever they were, longer ... if you thought that they had been through a traumatizing experience? Or would you attend to them quicker, you know? I think you would. (RN)

...I think one of the other things to consider is that while this study will help...both leaders and providers in the NICU... it is probably equally important to educate folks that are outside of these units because for resources and everything else, they're like, well, do they really need that? [They're] just sitting in the isolette or whatever. They're just a 
small baby; they just need to grow some or two years from now they won't remember any of this... (MD)

I would say that most people would think the trauma part is over. It's the, you know, the delivery's the traumatic part and we're just getting 'em after that and...we may...you know, we might be just continuing the trauma. (RN)

...it's going to impact them for a lifetime, right? Even if they don't recognize it, it will... (Dietician)

\section{And Now You Have Broken Them Too}

The potential undesirability of describing the infant's experience in the NICU as

traumatic focused on the implications of the term relative to the healthcare providers and parents, in addition to infants. For some healthcare providers, considering the NICU infant experience as a trauma is uncomfortable because their intention is to save lives and improve health. When providers considered how parents may perceive their infant's experience as a trauma, perceptions were mixed.

Perceptions of self:

...we would be okay saying that it's a traumatic experience for parents but to make that transition to the patient, it's hard for us to see because that's not what we intend at all. (Respiratory therapist)

...there's so many things at play like, you know, but I think we're already doing....as good a job that we can, like with the knowledge that we have. (RN)

...staff people wouldn't be happy about that word because it would insinuate that we aren't doing a good job. (RN)

...what kind of liability would that involve? Like if we're saying that we are traumatizing their baby, I could see people like having issue with that, right? (Dietician)

Perceptions of parents:

I think we use the word trauma, you know, quite a bit when we refer to the parents. You've been through a traumatic experience. This is traumatic for you. But we don't really use that word in reference to the baby. We don't talk about the baby having a traumatic experience. And they like that... but when you talk about their baby having a traumatic experience... now you are making things scarier for them. (RN) 
... I know it sounds kind of like we're, you know, putting the cape up and trying to pretend that there's not something there but, you know, I think we can acknowledge that it's hard. We can acknowledge that it is extremely stressful. We can acknowledge what we're doing and how things can be better. But we also have to be cognizant of what the family is going through at the time, you know, sometimes they are at a point where you throw one more thing on top of the pile and...now you've broken them too. (MD)

I think the parents are going through a trauma and they respond like, you know, PTSD in some situations. I think people recognize that as a thing but that's a parent's experience... maybe the baby's experience but I wouldn't want to call it that at this point. (MD)

\section{Perceptions of NICU Experience Change over Time}

Provider perceptions of the NICU environment and general patient care transitioned with increased work experience. Clinicians reflected on their novice encounter to the NICU with awe and excitement for the technical work, while as experts, clinicians expressed environmental desensitization and difficulty appreciating the fear of parents and families.

Providers as novices:

...when you come to the NICU you get to see a little bit of everything and it's like the purest form of pathophysiology. (MD)

I was a new grad so I thought it was exciting. (RN)

...stepping into the NICU and actually being at the bedside was much more technologic than I had anticipated. So I felt very overwhelmed...the whole environment itself was intimidating to me. (RN)

I was frightened. Yeah, very intimidated by the physical appearance of the infants. You know, just these weren't necessarily what I envisioned babies to look like... (Dietician)

Providers as experts:

...nothing surprises me anymore...you lose that perspective when you do it day in and day out for this many years. I think of how in awe I was of it when I started and now it's... it's just what we do. (RN)

...you do get comfortable...it's easy to forget the parents...have empathy for the parents. $(\mathrm{RN})$

...there's a lot to be said for gaining experience and gaining a level of comfort and confidence....you come in and you're learning those new things and you're just so 
focused on what does that alarm mean, how do I silence that, how do I hang this medication...I couldn't focus enough on really what the baby needed...I can't imagine what some babies feel...they come out here and it's traumatic. (RN)

The medicine part is still fun but there's a lot more things that make it un-fun that we deal with. (MD)

I have learned to detach myself. (MD)

\section{Trauma in the NICU: Whose Trauma Is It?}

The potential implications of using the term "trauma" involve infants, their parents and also clinicians. Clinicians describe their work in the NICU as rewarding and humbling; yet, clinicians themselves may suffer from vicarious traumatization as a result of their professional responsibilities and bearing witness to the suffering of the patient and family.

...we were in a full code and the baby had multiple anomalies...we were unable to resuscitate the baby but I turned around and I didn't know that the mom was not under and I'll never forget it...I couldn't imagine being that mom watching this experience, and we were just in action mode, just trying to act very quickly... we were focusing on the baby...I'll never forget that gut wrenching feeling inside... like I had impacted this poor mother...that was very traumatic to me... it was nine o'clock in the morning so you come back down to work...it's hard. (RN)

I had a patient...27 weeker with NEC. I was conducting a study at that time and I was gonna approach the mom about participating in the study. And I walked into the room and I saw the mom, I recognized her and she recognized me, it was a little bit tough to take because almost 2 years to the week I had gotten her first 27 weeker who died of NEC totalis and I eventually had to tell her that her second one had died of NEC totalis. (MD)

I was in charge...we had 4 very very sick critical deliveries happen at the same time...we just physically didn't have enough people to do what we had to do...I really felt like someone was going to die that day because I couldn't figure out how to make this work...I still dream about it sometimes... when you lived truly unsafe... it was very traumatic. (RN)

...I think we all suffer from that trauma...we dream about kids...especially the sick kids...I can't get them....get them out of my head... (MD)

\section{Not Knowing the Infant and Family Experience}


Clinicians enter healthcare to help others. Without proper training, however, in socialemotional-psychological aspects of care, misunderstandings may influence outcomes. The inability to accurately interpret differential behavioral responses to extremes of pain and stress, for both the infant and family, has significant implications that clinicians may not 'know' infants as well as they may think they do.

I can say very clearly... I did not get any [social-emotional training]...I thought that was for some other profession, social and other stuff was not medical. (MD)

It may feel traumatic to them but for us it's like whatever. $(\mathrm{RN})$

Kind of makes me most amazed... whatever we do...they care for a little bit...but then they settle down to that new norm...you put a chest tube in... you intubate them...painful and uncomfortable stuff and they just kind of take it in stride and kind of get used to it and I always think, well, how's this baby comfortable with all of that...some of them protest but the majority of them kind of accept it like, okay, this is what it is...they get more mature, they seem to mind a bit more but less mature ones are like do what you do. (MD)

...the perception was that, you know, there were bonding issues and this family was really standoffish...the mom was just scared. I mean she was just petrified. She would come into the unit just... and totally freeze. And because I knew her I could see that in her face; I knew her. But that was not folks' perception at all. And that was kind of an eye-opener, you know, just like wow, how many other moms or families do we judge that way, right? (Dietician).

\section{DISCUSSION}

Emergent themes from the focus groups describe the fragility of the infants, parents and clinicians themselves in the NICU environment with raw clarity. Despite their private acknowledgement of perceived trauma in the NICU, NICU clinicians remain reluctant to label the lived infant experience traumatic. Participants struggled to identify a word that exemplifies their perceptions of the infant experience. The term stressful was suggested as an alternative to trauma, largely because "trauma is such a loaded word" (MD). The study participants discussed common understandings of "trauma," including sexual and physical abuse, neglect, war, natural 
disaster, death and serious accidents. Three explicit and implicit concerns resonated among the focus groups:

- What does this term mean for infants?

- What does this term mean for parents?

- What does this term mean for clinicians?

\section{What does this term mean for infants?}

While clinicians expressed their perceptions of the infant experience as unpredictable, overstimulating, painful and stressful, they are reluctant to label the NICU hospitalization traumatic. An evolving understanding of the potential trauma of the NICU hospitalization is discussed by Sanders and Hall who propose the disruption of biologically embedded social connectedness is traumatic for NICU infants and their parents ${ }^{12}$. Preventing or mitigating trauma in the NICU thus requires the ongoing presence of co-regulatory parents, family and other attuned adult caregivers who understand that newborns and young infants communicate their feeling states and needs through their autonomic nervous systems and behaviors.

This communication system was recognized by pioneers including Drs. T. Berry Brazelton and Heildelise Als who together developed the Neonatal Behavioral Assessment Scale (NBAS) ${ }^{13,14}$. Subsequently, Dr. Als pioneered the Newborn Individualized Developmental Care and Assessment Program (NIDCAP). NIDCAP recognizes the evolutionarily-determined econiche of the fetus/preterm infant is the mother's womb. Thus, preterm birth displaces the newborn from the environment that meets his/her biologic expectancies and instead places the newborn in a modern NICU. During the typical NICU hospitalization, the newborn and parent experience ongoing separation, unpredictability and a suboptimal physical environment for preterm or sick infants. The NIDCAP Assessment Tool enables caregivers to collect information on the newborn autonomic states and behaviors that are then used to craft caregiving approaches 
that meet the infant's individualized needs. The goal of individualized developmental care is continuous assurance of protection, predictability, restfulness, intimate contact, pleasure and contentment ${ }^{15}$.

Research demonstrates improved outcomes for newborns cared for in NICUs that practice NIDCAP. A 2013 meta-analysis demonstrated significant reductions in ventilator days, length of stay, growth problem and severity of bronchopulmonary dysplasia. There were improvements in neurobehavioral functions up to 8 years, EEG coherence and frontal lobe/internal capsule white matter development ${ }^{16}$.

Comments by focus group participants who interpret these autonomic responses as tolerance of the stressful experience, rather than a traumatic surrender, suggest a significant need for an enhanced appreciation of infant autonomic reactivity and behavior. For example, the infant who rests in co-regulatory kangaroo care with a felt sense of safety may transition quickly to sympathetic arousal when disrupted for a procedure. Ideally, the procedural stress is brief, the infant is supported, and returns quickly to baseline autonomic stability. However, if the painful procedure is prolonged and/or support is not available, after exhausting all reserve, the infant may collapse into a "vegetative" energy-conserving mode with poor tone, listlessness and perhaps central apnea. An enhanced understanding of infant behavior and mitigation of disruptions of key caregiving relationships with implementation of trauma-informed care principles will result in improved short- and long-term outcomes for NICU infants and their parents.

\section{What does this term mean for parents?}

While most participants themselves have used or heard others describe the NICU parent experience as traumatic, many were concerned how parents might interpret their infants' 
experience being described as traumatic. Clinicians speculated that referencing "trauma" may compound parent worry and stress, potentially to toxic levels. Some believed it would cause parents to feel more helpless in their situation.

Prior research confirms that mothers of NICU infants experience acute stress disorder and PTSD more frequently than mothers of full term infants. Acute stress disorder may be seen at baseline in $18 \%$ of NICU mothers of preterm infants and PTSD in up to $30 \%$ of these mothers at one-month follow-up ${ }^{17}$. Mothers of preterm vs control infants experienced higher rates of depression, anxiety and traumatic symptoms over 14 months post-partum. Furthermore, as opposed to mothers of full-term infants, mothers of preterm infants reported no diminution of their traumatic symptoms from 14 days to 14 months after delivery ${ }^{18}$. Cheng et al (2016) reported a higher incidence of postpartum depression in fathers of preterm infants than fathers of term infants. Postnatal depression in fathers, not mothers, predicted lower cognitive performance in the child at 2 years of age ${ }^{19}$.

In an interpretive descriptive study of parents of preterm infants, Lasiuk et al described preterm birth as a "traumatic event that shattered parents' taken-for-granted expectations of parenthood" ${ }^{20}$. In their study, all parents agreed "stress" does not adequately describe their experience while the term "trauma" does ${ }^{20}$. Though needing confirmation with larger studies, their work calls the question - does using the word "trauma" to describe the parent and infant experience potentially harm parents psychologically? Or, alternatively does giving name to parents' experiences validate them and confirm that their health care providers understand their distress?

The Family Nurture Intervention (FNI) utilizes comprehensive NICU strategies to promote a calming environment for restoration of maternal-infant co-regulation. Promising 
findings include improved quality of maternal caregiving in the NICU, reduced maternal depression and anxiety at 4 months corrected age and improved neurodevelopmental outcomes of preterm infants at 18 months ${ }^{21-23}$. Adopting terminology that more accurately describes the NICU parent experience may open the door for more consistent utilization of evidence-based strategies, such as FNI, to mitigate the trauma experience of the infant and family.

\section{What does this term mean for clinicians?}

Despite describing their perceptions of the infant experience in the NICU with raw emotional language, the participant providers were reluctant to label the infant experience "traumatic." Clinicians expressed concerns that labeling the infant experience traumatic implied

they, as caregivers, were agents of trauma. Does provider intention change the experience of the infant? For some, the word "trauma" was perceived as a personal attack on their skills or intentions. Providing evidence-based practices to mitigate the impact of infant pain/stress including skin-to-skin, sucrose, and when necessary pain medications can relieve discomfort and assure all adult caregivers are addressing pain and stress.

Clinicians also shared their own difficulties processing or dealing with clinical experiences of loss, often because of workplace demands. Both primary and secondary traumatic stresses are recognized as adverse outcomes of providing intensive care services in health care providers. A survey of NICU and pediatric intensive care providers suggested that up to $17 \%$ had diagnosable post-traumatic stress disorder (PTSD) and another 66\% had concerns that were worrisome but did not reach criteria for diagnosis of PTSD. Provider resilience was inversely related with clinician PTSD symptoms ${ }^{24}$.

The focus group participants had significant NICU experience, and more than $80 \%$ reported having experienced a traumatic event. Participant comments about learning to "detach 
oneself" suggest vicarious traumatization in the NICU is associated with withdrawal of emotional responsiveness. Strategies for providing "neuroprotective care" for the neonatal clinician include debriefings, chaplaincy involvement, and peer support programs training other clinicians to provide emotional support for first-responders ${ }^{25-27}$. An enhanced understanding of the nature of traumatic experience for neonatal clinicians, both workplace and non-workplace related, may assist in developing strategies to reduce clinician traumatization and retraumatization while promoting effective caregiver-patient and family empathy.

The present study has several limitations. First, a larger sample of NICU clinicians will allow a richer description of clinician perception of the infant experience. Second, the focus group moderator was also a clinician in this NICU, and this may have influenced how openly clinicians shared their perceptions. Third, these findings may not be generalizable to those clinicians currently practicing trauma-informed NICU care, as those clinicians may perceive the infant experience differently than this cohort. These findings may also not apply to clinicians practicing in less acute environments.

Considering the range of infant experiences requires a new paradigm of NICU care that prioritizes social connectedness by optimizing parent-infant engagement from admission to discharge and beyond. Single-family room NICUs are a partial answer to maintaining physical proximity to the infant. However, for families and staff to fully support the NICU infant also requires establishing and maintaining emotional proximity. Only in both physical and emotional proximity will the parent or other adult caregiver begin to recognize and interpret the infant's autonomic regulatory and behavioral communications.

Making explicit the potential trauma of neonatal intensive care hospitalization and the healing power of social connectedness empowers professionals to provide evidence-based 
trauma-informed care practices. Recognizing and promoting parents as the experts in their infant's needs, better understanding the language of infant communication, and individualizing care to the needs of each infant and family are the foundation of a philosophy of care that will look different for each newborn and family. However vast the needs may seem -- the work is done incrementally-- one baby, one family, and one provider at a time.

\section{Acknowledgements:}

The authors would like to thank the NICU clinicians who generously participated in this study. No funding was received for this work 


\section{REFERENCES}

1. Perry BDP, R. Homeostasis, stress, trauma, and adaptation. A neurodevelopmental view of childhood trauma. Child and adolescent psychiatric clinics of North America. 1998;7(1):33-51.

2. Felitti V, Anda R, Nordenberg D, et al. Relationship of childhood abuse and household dysfunction to many of the leading causes of death in adults: The adverse childhood experiences (ACE) study. American Journal of Preventive Medicine. 1998;14(4):245258.

3. National Scientific Council on the Developing Child. Excessive Stress Disrupts the Architecture of the Developing Brain: Working Paper 3. 2014.

4. National Scientific Council on the Developing Child. Young Children Develop in an Environment of Relationships: Working Paper 1. 2004.

5. Pynoos RS, Steinberg AM, Layne CM, et al. Modeling constellations of trauma exposure in the National Child Traumatic Stress Network Core Data Set. Psychological Trauma: Theory, Research, Practice, and Policy. 2014;6(Suppl 1):S9-S17.

6. Coughlin ME. Transformative nursing in the NICU: trauma-informed age-appropriate care. New York, NY: Springer Publishing Company; 2014.

7. D'Agata AL, Sanders M, Grasso DJ, Young EE, Cong X, McGrath JM. Unpacking the burden of care for infants in the nicu. Advances in Nursing Science. 2016;39(3):244-256.

8. D'Agata AL, Young EE, Cong X, Grasso DJ, McGrath JM. Infant Medical Trauma in the Neonatal Intensive Care Unit (IMTN): A Proposed Concept for Science and Practice. Adv Neonatal Care. 2016;16(4):289-297.

9. Coughlin M. Trauma-informed care in the NICU: Evidence-based practice guidelines for neonatal clinicians. New York, NY: Springer Publishing Company; 2016.

10. Krueger RC, MA. Focus Groups: A Practical Guide for Applied Research. 4th ed. Thousand Oaks: CA: Sage Publications, Inc.; 2009.

11. Braun VC, V. Using thematic analysis in psychology. Qualitative Research in Psychology. 2006;3(2):77-101.

12. Sanders MR, Hall SL. Trauma-informed care in the newborn intensive care unit: promoting safety, security and connectedness. Journal Of Perinatology. 2017.

13. Als H, Tronick, E., Lester, B. M., \& Brazelton, T. B. The brazelton neonatal behavioral assessment scale (BNBAS). Norwell, MA1977.

14. Brazelton TB. Neonatal behavioral assessment scale. Philadelphia: J. B. Lippencott; 1973.

15. Als HG, L. Developmentaly supportive care in the neonatal intensive care unit. Zero to Three. 1995;15(6):2-11.

16. Ohlsson A, Jacobs SE. NIDCAP: A Systematic Review and Meta-analyses of Randomized Controlled Trials. Pediatrics. 2013;131(3):e881.

17. Shaw RJ, Bernard RS, DeBlois T, Ikuta LM, Ginzburg K, Koopman C. The Relationship Between Acute Stress Disorder and Posttraumatic Stress Disorder in the Neonatal Intensive Care Unit. Psychosomatics. 2009;50(2):131-137.

18. Kersting A, Dorsch, M., Wesselmann, U., Lüdorff, K., Witthaut, J., Ohrmann, P., HörnigFranz, I., Klockenbusch, W., Harms, E., Arolt, V. . Maternal posttraumatic stress response after the birth of a very low-birth-weight infant. Journal of Psychosomatic Research. 2004;57:473-476. 
19. Cheng ER, Kotelchuck M, Gerstein ED, Taveras EM, Poehlmann-Tynan J. Postnatal depressive symptoms among mothers and fathers of infants born preterm: prevalence and impacts on children's early cognitive function. Journal of developmental and behavioral pediatrics. 2016;37(1):33-42.

20. Lasiuk G, Comeau T, Newburn-Cook C. Unexpected: an interpretive description of parental traumas' associated with preterm birth. BMC Pregnancy and Childbirth. 2013;13(Suppl 1):S13-S13.

21. Hane AA, Myers MM, Hofer MA, et al. Family Nurture Intervention Improves the Quality of Maternal Caregiving in the Neonatal Intensive Care Unit: Evidence from a Randomized Controlled Trial. Journal of Developmental \& Behavioral Pediatrics. 2015;36(3):188-196.

22. Welch MG, Halperin MS, Austin J, et al. Depression and anxiety symptoms of mothers of preterm infants are decreased at 4 months corrected age with Family Nurture Intervention in the NICU. Archives of Women's Mental Health. 2016;19(1):51-61.

23. Welch MG, Firestein MR, Austin J, et al. Family Nurture Intervention in the Neonatal Intensive Care Unit improves social-relatedness, attention, and neurodevelopment of preterm infants at 18 months in a randomized controlled trial. Journal of Child Psychology and Psychiatry. 2015;56(11):1202-1211.

24. Dalia C, Abbas K, Colville G, Brierley J. Resilience, post-traumatic stress, burnout and coping in medical staff on the paediatric and neonatal intensive care unit (P/NICU) - A survey. Archives of Disease in Childhood. 2013;98(Suppl 1):A26-A27.

25. Keene EA, Hutton N, Hall B, Rushton C. Bereavement debriefing sessions: an intervention to support health care professionals in managing their grief after the death of a patient. Pediatric Nursing. 2010;36(4):185-189.

26. Sharp CG. Use of the chaplaincy in the neonatal intensive care unit. Southern Medical Journal. 1991;84(12):1482-1486.

27. Health MDo. Psychological First Aid (PFA). Downloaded from http://www.health.state.mn.us/oep/responsesystems/pfa.html. 
Figure 1. Infant Medical Trauma in the NICU conceptual model. Reprinted with permission from Wolters Kluwer Health, Inc. 
Table 1. Sample demographics, N=17 (n)

Gender

$\begin{array}{ll}\text { Female } & 82 \%(14) \\ \text { Male } & 18 \%(3)\end{array}$

Age

$\begin{array}{ll}31-40 & 24 \%(4) \\ 41-50 & 41 \%(7) \\ 51-60 & 35 \%(6)\end{array}$

Highest level of education

$\begin{array}{ll}\text { Diploma } & 6 \%(1) \\ \text { Baccalaureate } & 35 \%(6) \\ \text { Masters } & 29 \%(5) \\ \text { MD } & 18 \%(3) \\ \text { MD/PhD } & 12 \%(2)\end{array}$

Years of NICU experience

$\begin{array}{ll}1-5 & 6 \%(1) \\ 6-10 & 18 \%(3) \\ 11-15 & 24 \%(4) \\ >15 & 53 \%(9)\end{array}$

Personally, have you ever experienced a traumatic event

Yes

No
$82 \%(14)$

$18 \%(3)$ 
Table 2. Focus Group Questions

Opening: $\quad$ 1. How long you have been a clinician/leader and how long have you worked at the hospital?

Introductory: $\quad 2$. Have you received education and training on the socio-emotional aspects of neonatal care over the course of your career?

Transition: $\quad 3$. When you think of a baby and his or her personal experience of being a patient in the NICU, what comes to mind?

Key questions: 4. What feelings come to mind?

5. Are there some specific words/phrases that come to mind when you think about his or her experience?

6. When you hear the word "stress" or "stressful", what does that word mean to you?

7. When you hear the word "trauma" or "traumatic", what does the word mean to you?

8. Have you ever had a work experience in the NICU that you would label as traumatic? If you are able, can you please share the experience?

9. If the word 'trauma' were used to describe a baby's experience in the NICU, how would that impact you as a clinician/leader? Might it change how you practice?

Ending questions: $\quad 10$. We want to learn from you how you believe clinicians/leaders may react if the NICU baby's experience is defined as a traumatic personal experience. Is there anything you believe is important around this topic that we haven't asked about? Is there anything else you would like to share? 
Table 3. Data Analysis Codes and Themes

\begin{tabular}{|l|l|}
\hline \multicolumn{1}{|c|}{ Codes } & \multicolumn{1}{|c|}{ Themes } \\
\hline $\begin{array}{l}\text { Laying on of many hands } \\
\text { Difficult to experience NICU care } \\
\text { Burden of experience } \\
\text { Baby's experience }\end{array}$ & At Our Mercy \\
\hline $\begin{array}{l}\text { Defining trauma } \\
\text { Reconsidering use } \\
\text { Benefit of using term } \\
\text { What trauma communicates }\end{array}$ & Trauma Defined and Redefined \\
\hline $\begin{array}{l}\text { Do no harm } \\
\text { Unintended consequences } \\
\text { Liability } \\
\text { Fear to face truth of experience }\end{array}$ & And Now You Have Broken Them Too \\
\hline $\begin{array}{l}\text { Intense and exciting work } \\
\text { More in tune with baby over time } \\
\text { Loss of empathy } \\
\text { Chaos }\end{array}$ & Perceptions of NICU Change over Time \\
\hline $\begin{array}{l}\text { Losing the dream } \\
\text { Loss of clinician naivety } \\
\text { Parent helplessness } \\
\text { Trauma permeates }\end{array}$ & Trauma in the NICU: Whose Trauma Is It? \\
\hline $\begin{array}{l}\text { Muted sensitivity } \\
\text { Human connection and disconnection } \\
\text { Knowledge deficits } \\
\text { Coping capabilities }\end{array}$ & Misunderstanding the Infant and Family Experience \\
\hline
\end{tabular}

